\title{
CONSUMO DE ESTIMULANTES POR LOS ESTUDIANTES UNIVERSITARIOS ¿SE USA O SE ABUSA?
}

\author{
Stimulant use in university students, Is it used or abused?
}

*Joel Ricardo Chávez-Gutiérrez. **Johny Williams Menjivar Chacón, **Andrea María Sánchez Cerrato, **Mario Román Murcia Tovar, **Manuel Pineda Fajardo.

\section{RESUMEN}

Estimulante, es aquella sustancia que acelera la actividad del sistema nervioso central (SNC) y las encontramos en diferentes presentaciones. Objetivo: Identificar si los estudiantes refieren consumir estimulantes. Determinar el tipo de estimulante y frecuencia de consumo a su vez conocer si saben de los efectos secundarios no deseados. Metodología: Estudio descriptivo, prospectivo de corte transversal, realizado en la Universidad Nacional Autónoma de Honduras en el Valle de Sula (UNAH-VS), del 1 de febrero al 30 de junio de 2013. La información se obtuvo mediante un instrumento de recolección de datos tipo cuestionario, aplicado de forma aleatoria a un total de 1,950 universitarios. Resultados: se identificaron $1510(77 \%)$ estudiantes de la muestra que refirieron consumir estimulantes en algún momento del año académico; con un predominio por el sexo femenino con 824 (55\%); el grupo de edad promedio de mayor consumo de estimulantes correspondió a los menores de 25 años con 1435 (95\%). El estimulante de mayor consumo fué el café 876 (58\%), seguido por las bebidas energéticas con 453 (30\%). El 25\% (378) desco-

\footnotetext{
*Catedrático Titular I de la Escuela Universitaria de las Ciencias de la Salud de la Universidad Nacional Autónoma de Honduras en el Valle de Sula. EUCS/UNAH VS

**Estudiante de $\mathrm{V}$ año de medicina de la Escuela de Universitaria de Ciencias de la Salud de la UNAH VS.

Dirigir correspondecia a: joelchavez_16@yahoo.com
}

noce los efectos colaterales no deseados y $1132(75 \%)$ restante que tiene conocimiento de los efectos aun así los sigue consumiendo. Conclusiones existe un alto índice de estudiantes universitarios que consumen estimulantes como café y/o bebidas energizantes, entre otras y lo hacen conociendo de los efectos secundarios no deseados y sus riesgos por consumo, porque gustan del efecto y/o porque tienen una forma accesible de mantenerse más tiempo despierto y/o activos.

\section{PALABRAS CLAVE}

Bebidas, Efectos adversos, Estimulantes del Sistema Nervioso Central.

\section{SUMMARY}

Stimulant is a substance that speeds up the central nervous system (CNS) and are found in different forms. Objective: To identify if student refer consuming simulants. To Determine the type of stimulant and frequency of consumption in turn if they know of unwanted side effects. Methodology: A descriptive, cross-sectional prospective study applied at the National Autonomous University of Honduras in the Valle de Sula (UNAH-VS) during 1 February to 30 June 2013 The information was obtained through a data collection instrument type questionnaire, randomly applied to a total of 1,950 university. Results: We identified 1510 (77\%) students in the sample who reported 
consuming stimulants at some time of the academic year; with a predominance in females with 824 (55\%); the average age group with the highest consumption of stimulants corresponded to those under 25 years with 1435 (95\%). The stimulating higher coffee consumption was 876 (58\%), followed by energy drinks with 453 (30\%). 25\% (378) ignores the remaining unwanted side effects and 1132 (75\%) having knowledge of the effects yet still consuming. Conclusions: There is a high rate of college students use stimulants like coffee and/or energy drinks, among others, and they do know of the unwanted side effects and risks for consumption because like the effect and/or because they have an accessible to stay awake longer and/or assets.

\section{KEYWORDS}

Beverages, Adverse effects, Central Nervous System Stimulants.

\section{INTRODUCCIÓN}

Diversos factores influyen en los estudiantes de la Universidad Nacional Autónoma de Honduras en el Valle de Sula (UNAH-VS), para tomar la decisión de consumir estimulantes, tales como: carga académica, traslape trabajo-estudio y la falta de organización. ${ }^{(1,2)}$

La OMS, define estimulante; como la droga que acelera la actividad del sistema nervioso central (SNC) provocando euforia, desinhibición, menor control emocional, irritabilidad, agresividad, menor fatiga, disminución del sueño, excitación motora, inquietud. ${ }^{(3)}$

Droga; es toda sustancia que introducida al organismo por cualquier vía de administración, produce alteración del funcionamiento natural del SNC en el individuo y crea dependencia ya sea psicológica, física o ambas.

En base a las definiciones anteriores enunciamos que los estimulantes más empleados, de uso común y fácil acceso son: bebidas energéticas (a base de taurina), pastillas inhibidoras del sueño (anfetaminas), cafeína, cigarrillos, alcohol, marihuana etc.

Bebida Energética: Producto que ingresó recientemente en el mercado de nuestro país y se comercializa como alimentos.(4) Está compuesta por diversas sustancias que en acción conjunta proveen un efecto de activación en el organismo. Entre sus principales componentes tenemos:

a) Glúcidos: grupo compuesto por la sacarosa, la glucosa; aminoácidos como la taurina, la cual tiene diversos efectos en la modulación y migración neuronal, regulación del volumen de la células y su osmolaridad. ${ }^{(5)}$

b) Guaraná: es una planta de las amazonas; la que tiene efectos como: la pérdida de peso, estimulación del SNC, mejora la memoria, disminución de la agregación plaquetaria $^{3}$ y protección gástrica. ${ }^{(6)}$

c) Metilxantinas: (cafeína y teofilina), las que provocan reacciones adversas como: palpitaciones, insomnio, cefalea, pirosis, náuseas, vómitos, aumento en la frecuencia de las deposiciones con cambio en la coloración de las heces. 
Cafeína: se encuentra en el café y el té. Ambas son bebidas muy consumidas. Contienen minerales y vitaminas como complejo $B$ y vitamina $C$ y E, potasio, magnesio y sodio. ${ }^{(4,6)}$ Potencia la neurotransmisión dopaminérgica en el cerebro, evento que podría explicar su uso y abuso. Los efectos de la cafeína son diversos: en el SNC como psicoestimulante que aumenta el estado de alerta, genera un efecto antidepresivo, ansiolítico y mejora la función cognitiva, reduce la sensación del cansancio y fatiga, mantiene el estado de vigilia. En otros efectos puede ser hepatoprotector. ${ }^{(7)}$ Los efectos adversos más frecuentes son palpitaciones, taquicardia, molestias gástricas, temblor fino, nerviosismo e insomnio. ${ }^{(6)}$

Tabaco: Es el principal causante de cáncer de pulmón y muerte a nivel mundial, siendo una amenaza para la salud pública, debido a la triple dependencia: química, psicológica y social. ${ }^{(8,9)}$ Datos de la OMS indican que el consumo de tabaco es la causa de la muerte de aproximadamente 4 millones de personas cada año, el equivalente a una persona cada 8 segundos. ${ }^{(10)}$

El inicio y la experimentación al tabaco comienza a los 11 años, la prevalencia se ubica a la edad de 14 a 18 años corresponde un $14.8 \% .^{(11,12)}$ Los efectos que provoca el tabaco son de carácter negativo, teniendo mayor relación con enfermedades cardiopulmonares y cerebrovasculares como: enfermedad coronaria, enfermedad cerebro vascular, enfermedades pulmonar obstructiva crónica (EPOC), bronquitis crónica, enfermedades gastrointestinales, cáncer de pulmón, cáncer de cavidad bucal, cáncer de laringe y vías respiratorias altas. La nicotina es mediador de neurotransmisores segregado como la dopamina, noradrenalina, vasopresina y B-endorfinas. Estas inducen efectos euforizantes, cognitivos, regulación de la memoria, concentración y apetito en el SNC, son factores que la vuelven más adictiva que la heroína, cocaína o el alcohol. La adicción es la necesidad fisiológica en fumadores frecuentes. ${ }^{(13)}$ Otras drogas (ilícitas); Las de mayor consumo en Honduras son la cocaína y la marihuana. ${ }^{(14)}$

Cocaína: Popular y comercialmente, se da el nombre de cocaína a las sales de la cocaína, (clorhidrato de cocaína y sulfato de cocaína), que son los dos productos más puros en el proceso de refinación de la coca. La vida media de 4 a 6 horas (dosis dependiente) y su excreción es por vía renal. Los efectos clínicos que pueden enumerar: vasoconstricción periférica, taquicardia, hipertensión arterial, midriasis, temblor fino y sudoración, todo ello por acción sobre los receptores alfa y beta-adrenérgicos. Hay un potente estimulo en el SNC por acción dopaminérgica y activación de las hormonas tiroideas. ${ }^{(15,16)}$ Incrementa la energía, disminuye el apetito, da insomnio, aumento del rendimiento intelectual y físico, así como hiperactividad motora y verbal. Dosis altas pueden causar alteraciones de la capacidad crítica y discriminativa; alucinaciones visuales como auditivas incluso convulsiones. ${ }^{(15)}$

Marihuana: Es la más referida entre los jóvenes, la consumen para aumentar el rendimiento académico. ${ }^{(1)}$ La marihuana, al igual que el alcohol, tiene efectos depresores y las consideraciones sobre sus efectos 
de relajación física y mental y el sentimiento de bienestar. ${ }^{(13)}$

La memoria a corto plazo se encuentra deteriorada, no se puede realizar tareas complejas que requieren planificación, comprensión y razonamiento, porque está francamente disminuida. Produce además interrupción en la continuidad del discurso y lenguaje monótono. Estas alteraciones ocurren en forma selectiva sobre la memoria. ${ }^{(16)}$

Modafinilo (Pastillas): Es la de más consumo, fármaco que está prescrito para tratar la narcolepsia, apnea obstructiva del sueño y trastornos del sueño en el trabajo nocturno. Aún en investigación. ${ }^{(17,18)}$ Las formas de modafinilo en donde su mecanismo es muy variado se enumeran: orexinérgica, histaminérgica y dopaminérgica. Se usa como un fármaco estimulante para aumentar las horas de vigilia y tener "más tiempo para estudiar"; estos fármacos se facilitan por amigos y prescripción médica. ${ }^{(2)}$

El objetivo de la investigación fue identificar si los estudiantes consumían estimulantes, determinar el tipo y la frecuencia de consumo. A su vez conocer si sabían de los efectos secundarios que se producen.

\section{MATERIALES Y MÉTODOS}

Se realizó un estudio descriptivo, de corte transversal, en tiempo prospectivo, durante un período de 5 meses (comprendido entre el 1 de Febrero al 30 de Junio de 2013) aplicado a estudiantes de la UNAH-VS, aplicando un instrumento de recolección de datos tipo cuestionario, no incluyente. El que se respondió voluntariamente previo consenti- miento informado de forma verbal, obteniendo una muestra de 1,950 de un aproximado de 19,000 estudiantes inscritos; la aplicación del cuestionario se realizó de forma aleatoria en todo el campus, por varios encuestadores independientes los que previo explicaban los conceptos de estimulante y droga. Los datos se analizaron mediante Epi-Info y el paquete estadístico de Excel 2010. Aplicando la media, moda y promedios.

\section{RESULTADOS}

De los 1950 estudiantes que fueron encuestados, 1510 (77\%) refirieron consumir estimulantes y 440 (23\%) no consumían. De los estudiantes que si consumían estimulante; 824 (55\%) eran del sexo femenino y 686 (45\%) eran masculinos.

De los estudiantes encuestados, 842 (43\%) eran menores de 20 años. Ver grafica $N^{\circ} 1$.

Grafica N¹: Rango de edades de los estudiantes que consumían estimulantes

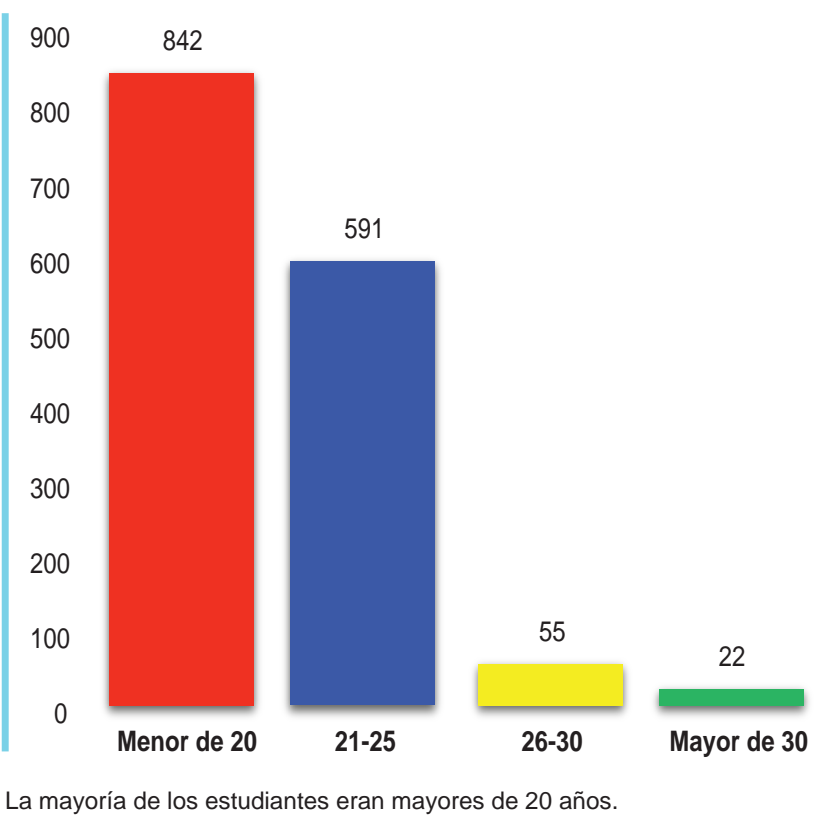


En relación a la jornada de estudio, se identificó que los que asistían durante el día consumían más estimulantes 1178 (78\%), que los de la jornada nocturna 332 (22\%).

Entre las razones por las que consumían estimulantes refirieron: "porque les gustaba el efecto causado" 649 (43\%), "se mantenían más horas despiertos" 378 (25\%), "se sentían estresados 196 (13\%), "se sentían menos fatigados" 181 (12\%) "lograban mayor concentración” 106 (7\%).

De los estudiantes universitarios que consumían estimulantes 378 (25\%) desconocían los efectos secundarios no deseados y 1132 (75\%) restante tenían conocimiento de los mismos y aun así los seguían consumiéndolo. Los estudiantes universitarios consumían diferentes sustancias para mantenerse estimulados. Ver grafica $\mathrm{N}^{\circ} 2$.

Grafica $\mathbf{N}^{\circ} 2$. Consumo de estimulantes

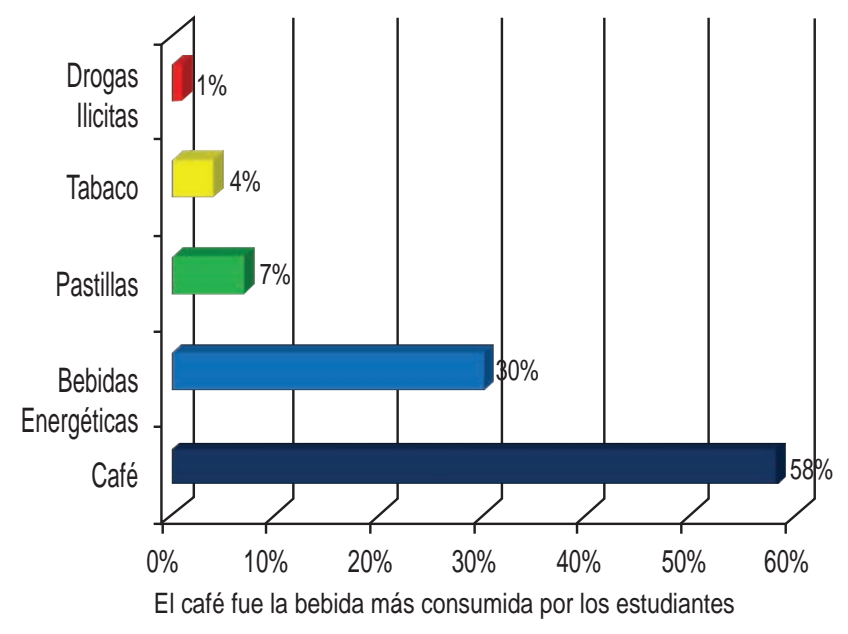

\section{DISCUSIÓN}

En la UNAH - VS, los estimulantes (sea el estimulante que sea y la administración que se prefiera) son utilizados por los estudiantes universitarios en un 77\% (1510 de 1950 entrevistas); las razones del uso son múltiples, justificando su abuso por que gustan de sus efectos colaterales, asumiendo los riesgos $(75 \%)$ porque son subestimados.

Además se identificó que 846 estudiantes (56\%) son jóvenes menores de 20 años y 589 (39\%) eran de edades entre 21-25 años. Este alto índice puede tener relación con el horario en que se llevaron a cabo las entrevistas (de $7 \mathrm{am}$ a $7 \mathrm{pm}$ ), donde se entrevistaron 1178 estudiantes (78\%) de las jornada diurna y 332 estudiantes (22\%) de la jornada nocturna donde la población es mayor de 26 años y generalmente trabaja de día. La literatura refiere que estas bebidas son más consumidas por jóvenes y que las empresas que las comercializan buscan su publicidad de persona a persona, auspiciando deportes extremos y a través de internet. ${ }^{(4,5)}$

Los resultados obtenidos tienen relación directa con el fácil acceso a las bebidas estimulantes que se puede relacionar con un inicio temprano de tabaquismo y alcoholismo, así como se refiere en la literatura. ${ }^{(10-12)}$ Identificando además, que las mujeres fueron las de mayor consumo (55\%).

Las bebidas estimulantes fueron consumidas en horas de la noche con fines de activar y estimular el SNC. Entre los estimulantes de mayor consumo tenemos: el café (58\%), bebidas energizantes (30\%), volviéndose ambos una opción de bajo costo para mantenerse despiertos y activos por más tiempo. Hecho que es preocupante, porque según refiere Lozano,(6) el consumo agudo o crónico de cafeína puede dar lugar a una 
amplia variedad de efectos adversos, intoxicaciones e incluso la muerte. Posee propiedades reforzadoras positivas, produce tolerancia y al cesar su consumo aparece un síndrome de abstinencia específico.

En conclusión, el uso de bebidas estimulantes es una práctica muy común en los estudiantes universitarios, siendo los jóvenes en su mayoria del sexo femenino los que más consumen, justificando su abuso porque les gusta los efectos colaterales, sin tomar en cuenta los riesgos.

Se recomienda hacer campañas de concientización sobre el consumo de las bebidas estimulantes, advertir de sus efectos secundarios sobre todo los efectos no deseados y regular la venta de estas bebidas en el campus.

\section{BIBLIOGRAFÍA}

1. Asencio P F, Cardemil MF, Betancour MP, Celedón PF, Martínez LF. Prevalencia y factores asociados al uso de drogas, fármacos y otras sustancias por estudiantes de medicina para aumentar el rendimiento académico. CIMEL [serial on the Internet]. 2009; 14(2): p. 1487-92. Disponible en:

http://revistas.concytec.gob.pe/scie lo. $p h p$ ? pd =S1680-83982009000 200003 \&script=sci_arttext.

2. Mazzoglio y Nabar MJ, Algieri RD, Dogliotti CB, Gazzotti AM, Jiménez-Villarruel HN, Rey LM. Utilización de sustancias psicoactivas en alumnos de anatomía y su implicación en el aprendizaje. Educación Médica [serial on the Internet]. 2011 Junio; 14(2): p. 129-132. Disponible en:

http://scielo.isciii.es/scielo.php?pid= S1575-18132011000200009\&script= sci_arttext.

http://www.educmed.net/sec/deAu tor. php?id=3a875a131444154255446\#.
3. Organización Mundial de la Salud. Serie de Informes Técnicos No 407. Comité de Expertos de la OMS en Farmacodependencia. Ginebra 1969. 16 informe. Dependencia y Abuso de Fármacos. Definiciones. Disponible en: http://whqlibdoc.who.int/trs/WHO_TRS _407_spa.pdf.

4. Cote Menéndez M, Rangel Garzón X, Sánchez Torres MY, Medina Lemus A. Bebidas energizantes ¿Hidratantes 0 Estimulantes?. Revista Facultad de Medicina de la Universidad Nacional de Colombia [serial on the Internet]. 2011 Sep; 59(3):256-262. Disponible en : http://www.scielo.org.co/pdf/rfmun/ v59n3/v59n3a08.pdf.

5. Roussos A, Franchello A, Flax Marco F, De Leo M, Larocca T, Barbeito S, et al. Bebidas energizantes y consumo en adolescentes. Revista SAN [serial on the Internet]. 2009 Jun; 10(2): p. 124127. Disponible en: 
http://www.revistasan.org.ar/pdf_files/ trabajos/vol_10/num_2/RSAN_10_ 2_124.pdf.

6. Pardo Lozano R, Álvarez García Y, Barral Tafalla D, Farré Albaladejo M. Cafeína: un nutriente, un fármaco, o una droga de abuso. ADICCIONES [serial on the Internet]. 2007; 19(3): p. 225-238.Disponible en:

http://www. adicciones.es/files/ediFa rre.pdf.

7. Tavares C, Kimiko Sakata R. Cafeína para el Tratamiento del Dolor. Rev Bras Anestesiol [serial on the Internet]. 2012; 62(3):387-401. Disponible en: http://www.scielo.br/pdf/rba/v62n3/es_ v62n3a11.pdf.

8. Prat Marín A, Fuentes Almendras M, Sanz Gallen R, Canela Argues R, Canela Soler J, Pardell A, et al. Epidemiología del tabaquismo en los estudiantes de ciencias de la salud; Rev. Saúde Pública [serial on the Internet]. 1994; 28(2):100-1006. Disponible en: http://actbr.org.br/uploads/conteu do/359_Epidemiologia_del_tabaquis mo_en_los_estudiantes_de_ciencias_ de_la_salud.pdf.

9. Vargas Pineda DR. Alcoholismo, Tabaquismo y Sustancias Psicoactivas. Rev. salud pública [serial on the Internet]. 2001 Mar; 3(1):74-88.

Disponible en: http://www.scielo.unal.edu.co/scie lo. php?script $=s c i$ arttext\&pid= s0124-00642001000100006\&lng=es $\& n r m=$.
10. Grupo tabaquismo. Tabaquismo. Arch. argent.pediatr. [serial on the Internet]. 2005; 103(5):464-475. Disponible en: http://www.scielo.org.ar/scielo.php?pid= s0325-00752005000500014\&script $=$ sci_arttext.

11. Revista de investigación del IHADFA VOI-2 2014 Disponible en: http://www. ihadfa.hn/index.php/revistade-investigaciones-del-ihadfa-vol2-2014.

12. Villena Ferrer A, Morena Rayo S, Párraga Martínez I, González Céspedes MD, Soriano Fernández $H$, López Torres Hidalgo J. Factores asociados al consumo de Tabaco en Adolescentes. REV CLÍN MED FAM [serial on the Internet]. 2009 Jun; 2 (7): 320-325. Disponible en:

http://scielo.isciii.es/scielo.php?pid= S1699-695X2009000 200002\&script=sci_arttext.

http://www.redalyc.org/pdf/1696/169 614509002.pdf.

13. González Marquetti T, Gálvez Cabrera E, Álvarez Valdés N, Cobas Ferrer FS, Cabrera del Valle N. Drogas y sexualidad: grandes enemigos. Rev Cubana Med Gen Integr [serial on the Internet]. 2005 Dic; 21(5-6). Disponible en: http://scielo.s/d.cu/scielo.php?script= sci_arttext\&pid=S0864- 21252005000 500015.

14. Rodríguez Funes GM, Brands B, Adlaf E, Giesbrecht N, Simich L, Miotto Wright MG. Factores de riesgo relacionados al uso de drogas ilegales: perspectiva críti- 
ca de familiares y personas cercanas en un centro de salud público en San Pedro Sula, Hondura. Rev. Latino-Am. Enfermagem [serial on the Internet]. 2009; 17(spe):796-802. Disponible en: http://www.scielo.br/scielo.php?script= sci_arttext\&pid=S0104-116920090007 00007.

15. Téllez Mosquera J, Cote Menéndez M. Efectos toxicológicos y neuropsiquiatricos producidos por consumo de cocaína. Rev Fac Med Univ Nac Colomb [serial on the Internet]. 2005; 53(1): 10-26. Disponible en: http://www.scielo.org.co/pdf/rfmun/v53n 1/v53n1a03.pdf.

16. Caballero Martínez L. ADICCIÓN A COCAÍNA: NEUROBIOLOGÍA, CLÍNICA, DIAGNÓSTICO Y TRATAMIENTO; (C) MINISTERIO DE SANIDAD Y CONSUMO CENTRO DE PUBLICACIONES (C)
SECRETARÍA GENERAL DE SANIDAD DELEGACIÓN DEL GOBIERNO PARA EL PLAN NACIONAL SOBRE DROGAS, España, 2005.

http://www.pnsd.msc.es/Categoria2/publica/pdf/AdiccionCocaina.pdf.

17. Sarubbo L. Alteraciones de la memoria producida por el uso de Marihuana. Rev Psiquiatr Urug [serial on the Internet]. 2009; 73(1):83-98.

Disponible en: http://www.spu.org.uy/revista/ago2009/03_RV_01.pdf

18. Olivares J, González Hernández J. Eficacia del modafinilo como potenciador cognitivo en sujetos sanos. Rev. Memoriza.com [serial on the Internet]. 2009; 2:39-43.

Disponible en: http://www.memoriza.com /documentos/revista/2009/modafinilo2009_2_39.pdf. 